\title{
Diastereoselective Aldol Reaction of $N, N$-Dibenzyl-a-amino Aldehydes with Ketones Catalyzed by Proline
}

\author{
Qiangbiao Pan ${ }^{1}$, Benli Zou ${ }^{1}$, Yuji Wang ${ }^{1}$ and Dawei $\mathrm{Ma}^{2} *$ \\ ${ }^{1}$ Department of Chemistry, Fudan University, Shanghai 200433,China, ${ }^{2}$ State Key \\ Laboratory of Bioorganic and Natural Products Chemistry, Shanghai Institute of \\ Organic Chemistry, Chinese Academy of Sciences, 354 Fenglin Lu, Shanghai 200032, \\ China.
}

\section{Supporting Information}


Typical experimental procedure. A suspension of $(S)$ - or $(R)$-proline $(29 \mathrm{mg}$, $0.25 \mathrm{mmol})$ and an aldehyde $(1.0 \mathrm{mmol})$ in $10 \mathrm{~mL}$ of acetone (entries $1-5$ in Table 1 and entries 1-6 in Table 2), or in $10 \mathrm{~mL}$ of acetone/DMSO (1:4) (entry 6 in Table 1), or in $10 \mathrm{~mL}$ of cyclopentanone/DMSO (1:4) (entries 7-10 in Table 2), or in $10 \mathrm{~mL}$ of hydroxyacetone/HMPA (1:4) (entries 11-16 in Table 2), was stirred at room temperature for 1-6 days. The reaction mixture was partitioned between aqueous saturated $\mathrm{NH}_{4} \mathrm{Cl}$ and ethyl acetate. The organic layer was separated and the aqueous layer was extracted. The combined organic layers were washed with brine, dried over $\mathrm{Na}_{2} \mathrm{SO}_{4}$, and concentrated. The residue was purified by flash chromatography eluting with hexane/ethyl acetate to give aldol product.

(4R,5S)-4-Hydroxy-6-phenyl-5-(tritylamino)hexan-2-one $2 \mathrm{a} \cdot[\alpha]_{\mathrm{D}}{ }^{17}+46.2(c$ $\left.1.1 \mathrm{CHCl}_{3}\right) .{ }^{1} \mathrm{H} \mathrm{NMR}\left(400 \mathrm{MHz}, \mathrm{CDCl}_{3}\right) \delta$ 7.5. $(\mathrm{d}, J=7.8 \mathrm{~Hz}, 6 \mathrm{H}), 7.26-7.11(\mathrm{~m}, 12 \mathrm{H})$, $6.85(\mathrm{~d}, J=6.9 \mathrm{~Hz}, 2 \mathrm{H}), 3.43(\mathrm{~m}, 1 \mathrm{H}), 2.71(\mathrm{~m}, 1 \mathrm{H}), 2.68(\mathrm{dd}, J=5.9,16.4 \mathrm{~Hz}, 1 \mathrm{H})$ $2.60(\mathrm{dd}, J=5.9,13.7 \mathrm{~Hz}, 1 \mathrm{H}), 2.49(\mathrm{dd}, J=8.2,13.7 \mathrm{~Hz}, 1 \mathrm{H}), 2.38(\mathrm{dd}, J=2.3,16.4$ $\mathrm{Hz}, 1 \mathrm{H}), 2.03$ (s, 3H). ${ }^{13} \mathrm{C} \mathrm{NMR}\left(400 \mathrm{MHz}, \mathrm{CDCl}_{3}\right) \delta 209.4,146.8,139.2,129.2,128.9$, $128.5,127.9,126.5,126.3,70.9,67.9,58.6,45.8,37.0,30.6$. FT-IR $v($ thin film) 3467 $3085,3060,3028,2931,2858,1708,1596,1493,1448,1360,1319,1211,1162,1101$ 1064, 1032, $1002 \mathrm{~cm}^{-1}$. EI-MS m/z $372(\mathrm{M}-\mathrm{Ph})^{+}, 244,243,228,182,165,91$. HRMS calcd for $\mathrm{C}_{31} \mathrm{H}_{32} \mathrm{NO}_{2}(\mathrm{M}+\mathrm{H})^{+}$requires $m / z$ 450.2434, found $m / z$ 450.2425.

(1S,2R)-(1-Benzyl-2-hydroxy-4-oxopentyl)carbamic acid tert-butyl ester 2b. $[\alpha]_{\mathrm{D}}{ }^{19}+13.1\left(c 1.0 \mathrm{CHCl}_{3}\right) .{ }^{1} \mathrm{H} \mathrm{NMR}\left(400 \mathrm{MHz}, \mathrm{CDCl}_{3}\right) \delta 7.31-7.21(\mathrm{~m}, 5 \mathrm{H}), 4.57$ $(\mathrm{d}, J=9.2 \mathrm{~Hz}, 1 \mathrm{H}), 3.96(\mathrm{~m}, 1 \mathrm{H}), 3.83(\mathrm{~m}, 1 \mathrm{H}), 3.67$ (brs, 1H), $2.98(\mathrm{dd}, J=3.1,14.2$ 
$\mathrm{Hz}, 1 \mathrm{H}), 2.82(\mathrm{dd}, J=7.3,14.2 \mathrm{~Hz}, 1 \mathrm{H}), 2.65(\mathrm{~m}, 2 \mathrm{H}), 2.17(\mathrm{~s}, 3 \mathrm{H}), 1.35(\mathrm{~s}, 9 \mathrm{H}) .{ }^{13} \mathrm{C}$ NMR (400 MHz, $\left.\mathrm{CDCl}_{3}\right) \delta 210.0,155.9,137.8,129.6,128.5,126.5,79.7,69.8,55.0$ 46.7, 36.0, 30.9, 28.4. FT-IR v (thin film) 3476, 3368, 3065, 3028, 2983, 2932, 1709, $1687,1604,1520,1496,1444,1423,1392,1366,1320,1266,1249,1172,1088,1050$ $1021 \mathrm{~cm}^{-1}$. EI-MS m/z $308(\mathrm{M}+\mathrm{H})^{+}, 216,164,160,142,120,116,99,91,57,43$. HRMS calcd for $\mathrm{C}_{17} \mathrm{H}_{25} \mathrm{NO}_{4} \mathrm{Na}(\mathrm{M}+\mathrm{Na})^{+}$requires $\mathrm{m} / \mathrm{z} 330.1682$, found $\mathrm{m} / \mathrm{z} 330.1668$. (1S,2S)-(1-Benzyl-2-hydroxy-4-oxo-pentyl)carbamic acid tert-butyl ester 3b. $[\alpha]_{\mathrm{D}}{ }^{18}-27.3\left(c 1.0 \mathrm{CHCl}_{3}\right) .{ }^{1} \mathrm{H}$ NMR $\left(400 \mathrm{MHz}, \mathrm{CDCl}_{3}\right) \delta 7.30-7.18(\mathrm{~m}, 5 \mathrm{H}), 4.99$ $(\mathrm{d}, J=9.6 \mathrm{~Hz}, 1 \mathrm{H}), 4.01(\mathrm{~m}, 1 \mathrm{H}), 3.69(\mathrm{q}, J=8.2 \mathrm{~Hz}, 1 \mathrm{H}), 3.53(\mathrm{~d}, J=2.3 \mathrm{~Hz}, 1 \mathrm{H})$, $2.90(\mathrm{~d}, J=7.8 \mathrm{~Hz}, 2 \mathrm{H}), 2.69(\mathrm{dd}, J=10.1,18.3 \mathrm{~Hz}, 1 \mathrm{H}), 2.55(\mathrm{dd}, J=2.3,18.3 \mathrm{~Hz}$ 1H), $2.13(\mathrm{~s}, 3 \mathrm{H}), 1.41(\mathrm{~s}, 9 \mathrm{H}) .{ }^{13} \mathrm{C} \mathrm{NMR}\left(400 \mathrm{MHz}, \mathrm{CDCl}_{3}\right) \delta$ 210.6, 156.0, 138.3, $129.5,128.5,126.4,79.5,66.8,55.5,47.3,38.7,30.8,28.4$.

(1S,2R)-(1-Benzyl-2-hydroxy-4-oxopentyl)carbamic acid benzyl ester 2c. $[\alpha]_{\mathrm{D}}{ }^{16}+10.6\left(c 1.0 \mathrm{CHCl}_{3}\right) .{ }^{1} \mathrm{H} \mathrm{NMR}\left(400 \mathrm{MHz}, \mathrm{CDCl}_{3}\right) \delta 7.32-7.18(\mathrm{~m}, 10 \mathrm{H}), 5.01(\mathrm{~s}$, 2H), $4.87(\mathrm{~d}, J=8.2 \mathrm{~Hz}, 1 \mathrm{H}), 3.98(\mathrm{~m}, 1 \mathrm{H}), 3.89(\mathrm{~m}, 1 \mathrm{H}), 3.60(\mathrm{~s}, 1 \mathrm{H}), 2.99$ (dd, $J=$ 3.7, $13.7 \mathrm{~Hz}, 1 \mathrm{H}), 2.83(\mathrm{dd}, J=8.3,13.7 \mathrm{~Hz}, 1 \mathrm{H}), 2.64(\mathrm{~m}, 2 \mathrm{H}), 2.13(\mathrm{~s}, 3 \mathrm{H}) .{ }^{13} \mathrm{C} \mathrm{NMR}$ $\left(400 \mathrm{MHz}, \mathrm{CDCl}_{3}\right) \delta 209.9,156.3,137.5,136.5,129.6,128.63,128.60,128.2,128.0$ 126.6, 69.6, 66.8, 55.5, 46.6, 35.8, 30.9. FT-IR v (thin film) 3481, 3352, 3067, 3035, $2958,2925,2885,1709,1687,1604,1526,1495,1423,1353,1346,1322,1249,1087$ 1076, 1047, $1035 \mathrm{~cm}^{-1}$. ESI-MS m/z $342.2(\mathrm{M}+\mathrm{H})^{+}$. HRMS calcd for $\mathrm{C}_{20} \mathrm{H}_{24} \mathrm{NO}_{4}(\mathrm{M}$ $+\mathrm{H})^{+}$requires $m / z$ 342.1706, found $m / z$ 342.1689.

(1S,2S)-(1-Benzyl-2-hydroxy-4-oxopentyl)carbamic acid benzyl ester 3c. 
$[\alpha]_{\mathrm{D}}{ }^{17}-17.5\left(c 1.1 \mathrm{CHCl}_{3}\right) .{ }^{1} \mathrm{H}$ NMR $\left(400 \mathrm{MHz}, \mathrm{CDCl}_{3}\right) \delta 7.36-7.19(\mathrm{~m}, 10 \mathrm{H}), 5.30(\mathrm{~d}$, $J=10.1 \mathrm{~Hz}, 1 \mathrm{H}), 5.08(\mathrm{dd}, J=12.4,15.6 \mathrm{~Hz}, 2 \mathrm{H}), 4.02(\mathrm{~m}, 1 \mathrm{H}), 3.76(\mathrm{q}, J=8.2 \mathrm{~Hz}$ 1H), $3.51(\mathrm{~s}, 1 \mathrm{H}), 2.91(\mathrm{~d}, J=7.8 \mathrm{~Hz}, 2 \mathrm{H}), 2.65(\mathrm{dd}, J=10.1,18.3 \mathrm{~Hz}, 1 \mathrm{H}), 2.52(\mathrm{dd}, J$ $=2.3,18.3 \mathrm{~Hz}, 1 \mathrm{H}), 2.09$ (s, 3H). ${ }^{13} \mathrm{C} \mathrm{NMR}\left(400 \mathrm{MHz}, \mathrm{CDCl}_{3}\right) \delta 210.5,156.5,138.1$, $136.2,129.5,128.6,128.2,128.1,126.6,66.8,66.7,56.1,47.2,38.6,30.8$.

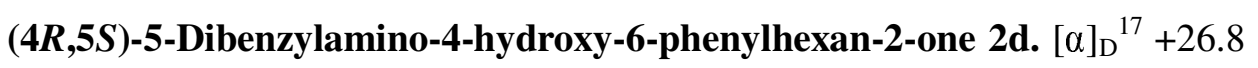
(c $\left.1.0 \mathrm{CHCl}_{3}\right) .{ }^{1} \mathrm{H}$ NMR $\left(400 \mathrm{MHz}, \mathrm{CDCl}_{3}\right) \delta$ 7.31-7.14 (m, 15H), $4.27(\mathrm{~m}, 1 \mathrm{H}), 3.73$ $(\mathrm{d}, J=13.8 \mathrm{~Hz}, 2 \mathrm{H}), 3.61(\mathrm{~d}, J=13.8 \mathrm{~Hz}, 2 \mathrm{H}), 3.07(\mathrm{dd}, J=7.3,14.2 \mathrm{~Hz}, 1 \mathrm{H}), 2.99$ $(\mathrm{dd}, J=5.5,14.2 \mathrm{~Hz}, 1 \mathrm{H}), 2.92(\mathrm{~m}, 1 \mathrm{H}), 2.76(\mathrm{dd}, J=2.3,17.9 \mathrm{~Hz}, 1 \mathrm{H}), 2.42(\mathrm{dd}, J=$ 13.6, 17.9 Hz, 1H), 2.05 (s, 3H). $\left.{ }^{13} \mathrm{C} \mathrm{NMR} \mathrm{(400} \mathrm{MHz,} \mathrm{CDCl}_{3}\right) \delta 210.4,141.3,139.9$, $129.7,128.9,128.4,128.3,127.0,126.0,68.4,62.9,54.7,48.2,32.4,30.8$. FT-IR $v$ (thin film) 3477, 3086, 3063, 3028, 2928, 2804, 1709, 1603, 1495, 1454, 1364, 1164 , 1123, 1095, 1074, $1029 \mathrm{~cm}^{-1}$. ESI-MS m/z $388.2(\mathrm{M}+\mathrm{H})^{+}$. HRMS calcd for $\mathrm{C}_{26} \mathrm{H}_{30} \mathrm{NO}_{2}(\mathrm{M}+\mathrm{H})^{+}$requires $m / z$ 388.2277, found $m / z$ 388.2299.

(4S,5S)-5-Dibenzylamino-4-hydroxy-6-phenylhexan-2-one 3d. $[\alpha]_{\mathrm{D}}{ }^{17}+20.2$ $\left(c 1.2 \mathrm{CHCl}_{3}\right) .{ }^{1} \mathrm{H} \mathrm{NMR}\left(400 \mathrm{MHz}, \mathrm{CDCl}_{3}\right) \delta 7.33-7.18(\mathrm{~m}, 15 \mathrm{H}), 4.04(\mathrm{~d}, J=13.3 \mathrm{~Hz}$, 2H), $4.00(\mathrm{~m}, 1 \mathrm{H}), 3.41(\mathrm{~d}, J=13.3 \mathrm{~Hz}, 2 \mathrm{H}), 3.12(\mathrm{dd}, J=3.7,12.8 \mathrm{~Hz}, 1 \mathrm{H}), 2.83-2.72$ (m, 2H), $2.51(\mathrm{dd}, J=8.7,16.5 \mathrm{~Hz}, 1 \mathrm{H}), 2.13(\mathrm{dd}, J=2.3,16.5 \mathrm{~Hz}, 1 \mathrm{H}), 2.01(\mathrm{~s}, 3 \mathrm{H})$. ${ }^{13} \mathrm{C} \mathrm{NMR}\left(400 \mathrm{MHz}, \mathrm{CDCl}_{3}\right) \delta 209.3,140.2,139.4,129.3,129.2,128.7,128.6,127.3$ $126.4,67.5,63.3,54.5,48.4,31.1,31.0$.

(4R,5S)-5-Dibenzylamino-4-hydroxyhexan-2-one 7a (containing about 3\% inseparable isomer 8a). $[\alpha]_{\mathrm{D}}{ }^{17}+45.2\left(\mathrm{c} 1.1 \mathrm{CHCl}_{3}\right) .{ }^{1} \mathrm{H} \mathrm{NMR}\left(400 \mathrm{MHz}, \mathrm{CDCl}_{3}\right)$ 
(major product 7a) $\delta$ 7.33-7.21 (m, 10H), $3.97(\mathrm{~m}, 1 \mathrm{H}), 3.72(\mathrm{~d}, J=13.7 \mathrm{~Hz}, 2 \mathrm{H}), 3.40$ $(\mathrm{d}, J=13.7 \mathrm{~Hz}, 2 \mathrm{H}), 3.03(\mathrm{dd}, J=1.8,17.8 \mathrm{~Hz}, 1 \mathrm{H}), 2.65(\mathrm{~m}, 1 \mathrm{H}), 2.37(\mathrm{dd}, J=8.7$ $17.8 \mathrm{~Hz}, 1 \mathrm{H}), 2.08(\mathrm{~s}, 3 \mathrm{H}), 1.16(\mathrm{~d}, J=6.9 \mathrm{~Hz}, 1 \mathrm{H})$. FT-IR $v$ (thin film) 3454, 3086, $3063,3029,2988,2932,2806,1708,1603,1586,1495,1454,1363,1263,1240,1164$, $1074,1062,1028 \mathrm{~cm}^{-1}$. ESI-MS m/z $312.2(\mathrm{M}+\mathrm{H})^{+}$. HRMS calcd for $\mathrm{C}_{20} \mathrm{H}_{26} \mathrm{NO}_{2}(\mathrm{M}+$ $\mathrm{H})^{+}$requires $m / z$ 312.1964, found $m / z 312.1965$.

$(4 R, 5 S)-5-D i b e n z y l a m i n o-4-h y d r o x y-6-m e t h y l h e p t a n-2-o n e \quad 7 b . \quad[\alpha]_{D}{ }^{17}$ $+10.3\left(\right.$ c $\left.1.0 \mathrm{CHCl}_{3}\right) .{ }^{1} \mathrm{H} \mathrm{NMR}\left(400 \mathrm{MHz}, \mathrm{CDCl}_{3}\right) \delta$ 7.34-7.22( m, 10H), $4.31(\mathrm{~m}, 1 \mathrm{H})$, $3.71(\mathrm{dd}, J=13.7,16.9 \mathrm{~Hz}, 4 \mathrm{H}), 3.10(\mathrm{~d}, J=6.0 \mathrm{~Hz}, 1 \mathrm{H}), 2.73(\mathrm{dd}, J=2.3,16.9 \mathrm{~Hz}$, 1H), $2.54(\mathrm{dd}, J=10.1,16.9 \mathrm{~Hz}, 1 \mathrm{H}), 2.42(\mathrm{t}, J=6.4 \mathrm{~Hz}, 1 \mathrm{H}), 2.20(\mathrm{~m}, 1 \mathrm{H}), 2.14(\mathrm{~s}$, $3 \mathrm{H}), 1.15(\mathrm{~d}, J=6.8 \mathrm{~Hz}, 3 \mathrm{H}), 1.05(\mathrm{~d}, J=6.8 \mathrm{~Hz}, 3 \mathrm{H}) .{ }^{13} \mathrm{C} \mathrm{NMR}\left(400 \mathrm{MHz}, \mathrm{CDCl}_{3}\right) \delta$ 210.0, 139.9, 129.1, 128.5, 127.2, 66.5, 65.6, 55.4, 48.4, 31.0, 26.7, 23.5, 20.2. FT-IR $v$ (thin film) 3497, 3086, 3063, 3029, 2956, 2803, 1707, 1603, 1586, 1495, 1419, 1361, 1291, 1238, 1209, 1165, 1106, 1069, $1029 \mathrm{~cm}^{-1}$. EI-MS m/z $296(\mathrm{M}-\mathrm{Pr}-i)^{+}$, 253, 252, 181, 91, 65, 43. HRMS calcd for $\mathrm{C}_{22} \mathrm{H}_{30} \mathrm{NO}_{2}(\mathrm{M}+\mathrm{H})^{+}$requires $m / z, 340.2277$, found $m / z 340.2266$.

(4S,5S)-5-Dibenzylamino-4-hydroxy-6-methylheptan-2-one 8b. $[\alpha]_{D}{ }^{17}-24.4$ (c $\left.0.5 \mathrm{CHCl}_{3}\right) .{ }^{1} \mathrm{H}$ NMR (400 MHz, $\left.\mathrm{CDCl}_{3}\right) \delta$ 7.39-7.22 ( m, 10H), $4.21(\mathrm{~m}, 1 \mathrm{H}), 3.98$ $(\mathrm{d}, J=13.3 \mathrm{~Hz}, 2 \mathrm{H}), 3.54(\mathrm{~d}, J=13.3 \mathrm{~Hz}, 2 \mathrm{H}), 2.46(\mathrm{dd}, J=9.6,16.0 \mathrm{~Hz}, 1 \mathrm{H})$, 2.32-2.22(m, 3H), $2.12(\mathrm{~s}, 3 \mathrm{H}), 1.08(\mathrm{~d}, J=6.9 \mathrm{~Hz}, 3 \mathrm{H}), 1.05(\mathrm{~d}, J=6.9 \mathrm{~Hz}, 3 \mathrm{H}) .{ }^{13} \mathrm{C}$ NMR (400 MHz, $\left.\mathrm{CDCl}_{3}\right) \delta 209.1,139.5,129.5,128.5,127.2,65.8,54.7,49.3,31.0$ $26.1,23.6,20.2$. 
(4R,5S)-5-Dibenzylamino-4-hydroxy-7-methyloctan-2-one 7 c. $[\alpha]_{\mathrm{D}}^{18}+10.2$ (c $\left.1.0 \mathrm{CHCl}_{3}\right) .{ }^{1} \mathrm{H} \mathrm{NMR}\left(400 \mathrm{MHz}, \mathrm{CDCl}_{3}\right) \delta$ 7.32-7.20 (m, 10H), $4.28(\mathrm{~m}, 1 \mathrm{H}), 3.65$ $(\mathrm{dd}, J=13.3,16.5 \mathrm{~Hz}, 4 \mathrm{H}), 3.06($ br s $1 \mathrm{H}), 2.62(\mathrm{dd}, J=2.8,17.4 \mathrm{~Hz}, 1 \mathrm{H}), 2.54(\mathrm{~m}$, $1 \mathrm{H}), 2.50(\mathrm{dd}, J=9.2,17.4 \mathrm{~Hz}, 1 \mathrm{H}), 2.10(\mathrm{~s}, 3 \mathrm{H}), 1.87(\mathrm{~m}, 1 \mathrm{H}), 1.65(\mathrm{~m}, 1 \mathrm{H}), 1.25(\mathrm{~m}$, $1 \mathrm{H}), 0.92(\mathrm{~d}, J=6.4 \mathrm{~Hz}, 3 \mathrm{H}), 0.73(\mathrm{~d}, J=6.4 \mathrm{~Hz}, 3 \mathrm{H}) .{ }^{13} \mathrm{C} \mathrm{NMR}\left(400 \mathrm{MHz}, \mathrm{CDCl}_{3}\right) \delta$ 210.3, 140.3, 129.1, 128.4, 127.0, 67.6, 58.4, 54.8, 48.2, 35.5, 30.9, 25.3, 23.4, 22.8. FT-IR v (thin film) 3484, 3086, 3064, 3029, 2955, 2867, 2805, 1710, 1603, 1586, 1495 , 1467, 1454, 1365, 1276, 1164, 1071, $1029 \mathrm{~cm}^{-1}$. EI-MS m/z $354(\mathrm{M}+\mathrm{H})^{+}, 296,267$, 266, 181, 91, 65, 57, 43. HRMS calcd for $\mathrm{C}_{23} \mathrm{H}_{32} \mathrm{NO}_{2}(\mathrm{M}+\mathrm{H})^{+}$requires $m / z 354.2434$, found $m / z, 354.2424$.

(4S,5S)-5-Dibenzylamino-4-hydroxy-7-methyloctan-2-one 8c. $[\alpha]_{\mathrm{D}}{ }^{17}+1.4(c$ 1.6 $\left.\mathrm{CHCl}_{3}\right) .{ }^{1} \mathrm{H} \mathrm{NMR}\left(400 \mathrm{MHz}, \mathrm{CDCl}_{3}\right) \delta$ 7.32-7.21 (m, 10H), 4.18 (brs, $\left.1 \mathrm{H}\right), 3.96(\mathrm{~m}$, 1H), $3.90(\mathrm{~d}, J=13.3 \mathrm{~Hz}, 2 \mathrm{H}), 3.43(\mathrm{~d}, J=13.3 \mathrm{~Hz}, 2 \mathrm{H}), 2.52(\mathrm{dd}, J=9.2,15.6 \mathrm{~Hz}$, 1H), $2.49(\mathrm{~m}, 1 \mathrm{H}), 2.37(\mathrm{dd}, J=2.8,15.6 \mathrm{~Hz}, 1 \mathrm{H}), 2.14(\mathrm{~s}, 3 \mathrm{H}), 1.68(\mathrm{~m}, 1 \mathrm{H}), 1.58(\mathrm{~m}$ 1H), $1.28(\mathrm{~m}, 1 \mathrm{H}), 0.93(\mathrm{~d}, J=2.3 \mathrm{~Hz}, 3 \mathrm{H}), 0.92(\mathrm{~d}, J=2.3 \mathrm{~Hz}, 3 \mathrm{H}) .{ }^{13} \mathrm{C}$ NMR $(400$ $\left.\mathrm{MHz}, \mathrm{CDCl}_{3}\right) \delta 209.0,139.5,129.2,128.5,127.3,68.4,59.9,54.3,48.4,35.0,31.1$, $26.4,23.5,22.9$.

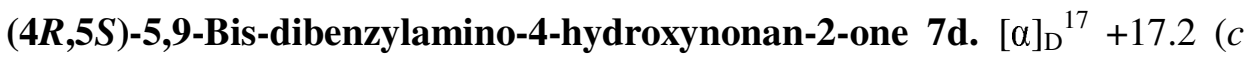
1.0 $\left.\mathrm{CHCl}_{3}\right) .{ }^{1} \mathrm{H}$ NMR $\left(400 \mathrm{MHz}, \mathrm{CDCl}_{3}\right) \delta$ 7.39-7.21 (m, 20H), $4.20(\mathrm{~m}, 1 \mathrm{H}), 3.63(\mathrm{dd}$, $J=13.7,17.9 \mathrm{~Hz}, 4 \mathrm{H}), 3.54(\mathrm{dd}, J=13.7,17.0 \mathrm{~Hz}, 4 \mathrm{H}), 2.60(\mathrm{dd}, J=2.3,17.8 \mathrm{~Hz}, 1 \mathrm{H})$, 2.47-2.35 (m, 4H), $2.08(\mathrm{~s}, 3 \mathrm{H}), 1.70-1.32(\mathrm{~m}, 6 \mathrm{H}) .{ }^{13} \mathrm{C} \mathrm{NMR}\left(400 \mathrm{MHz}, \mathrm{CDCl}_{3}\right) \delta$ $210.4,140.24,140.16,129.1,128.9,128.4,128.3,127.1,126.9,67.6,60.7,58.5,54.8$ 
53.3, 48.2, 30.9, 27.5, 26.0, 25.7. FT-IR v (thin film) 3484, 3086, 3063, 3028, 2934, 2858, 2799, 1710, 1602, 1494, 1453, 1364, 1247, 1162, 1128, 1074, $1028 \mathrm{~cm}^{-1}$. ESI-MS $m / z$, $549.3(\mathrm{M}+\mathrm{H})^{+}$. HRMS calcd for $\mathrm{C}_{37} \mathrm{H}_{45} \mathrm{~N}_{2} \mathrm{O}_{2}(\mathrm{M}+\mathrm{H})^{+}$requires $\mathrm{m} / z$ 549.3482, found $\mathrm{m} / \mathrm{z} 549.3471$.

(4S,5S)-5,9-Bis-dibenzylamino-4-hydroxynonan-2-one $8 \mathrm{~d} \cdot[\alpha]_{\mathrm{D}}{ }^{18}+10.3(c$ 1.25 $\left.\mathrm{CHCl}_{3}\right) .{ }^{1} \mathrm{H} \mathrm{NMR}\left(400 \mathrm{MHz}, \mathrm{CDCl}_{3}\right) \delta$ 7.40-7.22 (m, 20H), $3.89(\mathrm{~m}, 1 \mathrm{H}), 3.86(\mathrm{~d}$, $J=13.3 \mathrm{~Hz}, 2 \mathrm{H}), 3.56(\mathrm{dd}, J=13.7,16.8 \mathrm{~Hz}, 4 \mathrm{H}), 3.39(\mathrm{~d}, J=13.3 \mathrm{~Hz}, 2 \mathrm{H}), 2.46-2.33$ $(\mathrm{m}, 4 \mathrm{H}), 2.29(\mathrm{dd}, J=2.8,15.6 \mathrm{~Hz}, 1 \mathrm{H}), 2.11(\mathrm{~s}, 3 \mathrm{H}), 1.63-1.22(\mathrm{~m}, 6 \mathrm{H}) .{ }^{13} \mathrm{C} \mathrm{NMR}$ $\left(400 \mathrm{MHz}, \mathrm{CDCl}_{3}\right) \delta 208.8,140.1,139.3,129.3,128.9,128.6,128.3,127.3,127.0$ $67.9,62.2,58.6,54.4,53.1,48.4,31.1,27.8,26.4,25.8$.

\section{(4R,5S)-6-(4-Benzyloxyphenyl)-5-dibenzylamino-4-hydroxyhexan-2-one}

7e. $[\alpha]_{\mathrm{D}}{ }^{17}+31.0\left(c 1.0 \mathrm{CHCl}_{3}\right) .{ }^{1} \mathrm{H}$ NMR $\left(400 \mathrm{MHz}, \mathrm{CDCl}_{3}\right) \delta$ 7.47-7.15 (m, 15H), $7.12(\mathrm{~d}, J=8.5 \mathrm{~Hz}, 2 \mathrm{H}), 6.92(\mathrm{~d}, J=8.5 \mathrm{~Hz}, 2 \mathrm{H}), 5.08(\mathrm{~s}, 2 \mathrm{H}), 4.26(\mathrm{~m}, 1 \mathrm{H}), 3.72(\mathrm{~d}, J$ $=13.8 \mathrm{~Hz}, 2 \mathrm{H}), 3.61(\mathrm{~d}, J=13.8 \mathrm{~Hz}, 2 \mathrm{H}), 3.04-2.83(\mathrm{~m}, 4 \mathrm{H}), 2.75(\mathrm{dd}, J=2.3,17.6 \mathrm{~Hz}$, $1 \mathrm{H}), 2.40(\mathrm{dd}, J=9.6,17.6 \mathrm{~Hz}, 1 \mathrm{H}), 2.06(\mathrm{~s}, 3 \mathrm{H}) .{ }^{13} \mathrm{C} \mathrm{NMR}\left(400 \mathrm{MHz}, \mathrm{CDCl}_{3}\right) \delta 210.4$, $157.2,139.9,137.3,133.5,130.5,128.9,128.7,128.3,128.0,127.6,127.0,114.9,70.2$, 68.5, 63.0, 54.8, 48.3, 31.4, 30.8. FT-IR v (thin film) 3385, 3086, 3061, 3031, 2928 , $2809,1716,1610,1582,1511,1495,1453,1378,1297,1242,1117,1127,1024,1008$ $\mathrm{cm}^{-1}$. ESI-MS $m / z 494.3(\mathrm{M}+\mathrm{H})^{+}$. HRMS calcd for $\mathrm{C}_{33} \mathrm{H}_{36} \mathrm{NO}_{3}(\mathrm{M}+\mathrm{H})^{+}$requires $\mathrm{m} / z$ 494.2696, found $m / z$ 494.2735.

(4S,5S)-6-(4-Benzyloxyphenyl)-5-dibenzylamino-4-hydroxyhexan-2-one 8e. $[\alpha]_{\mathrm{D}}{ }^{17}+32.1\left(c 1.0 \mathrm{CHCl}_{3}\right) .{ }^{1} \mathrm{H} \mathrm{NMR}\left(400 \mathrm{MHz}, \mathrm{CDCl}_{3}\right) \delta$ 7.44-7.22 (m, 15H), $7.09(\mathrm{~d}$ 
$J=8.5 \mathrm{~Hz}, 2 \mathrm{H}), 6.91(\mathrm{~d}, J=8.5 \mathrm{~Hz}, 2 \mathrm{H}), 5.06(\mathrm{~s}, 2 \mathrm{H}), 4.13(\mathrm{brs}, 1 \mathrm{H}), 4.02(\mathrm{~d}, J=13.3$ $\mathrm{Hz}, 2 \mathrm{H}), 3.97(\mathrm{~m}, 1 \mathrm{H}), 3.41(\mathrm{~d}, J=13.3 \mathrm{~Hz}, 2 \mathrm{H}), 3.06(\mathrm{~m}, 1 \mathrm{H}), 2.72(\mathrm{~m}, 2 \mathrm{H}), 2.48(\mathrm{dd}$, $J=9.2,16.5 \mathrm{~Hz}, 1 \mathrm{H}), 2.15(\mathrm{dd}, J=2.8,16.5 \mathrm{~Hz}, 1 \mathrm{H}), 2.02(\mathrm{~s}, 3 \mathrm{H}) .{ }^{13} \mathrm{C}$ NMR $(400$ $\left.\mathrm{MHz}, \mathrm{CDCl}_{3}\right) \delta 209.3,157.4,139.4,137.2,132.4,130.3,129.2,128.7,128.6,128.1$ $127.6,127.3,115.2,70.2,67.5,63.4,54.5,48.5,31.0,30.3$.

(4R,5S)-5-Dibenzylamino-4-hydroxy-6-methoxymethoxyhexan-2-one $7 f$. $[\alpha]_{\mathrm{D}}{ }^{17}+43.0\left(c 1.0 \mathrm{CHCl}_{3}\right) .{ }^{1} \mathrm{H} \mathrm{NMR}\left(400 \mathrm{MHz}, \mathrm{CDCl}_{3}\right) \delta$ 7.33-7.21 (m, 10H), $4.67(\mathrm{~s}$, 2H), $4.26(\mathrm{~m}, 1 \mathrm{H}), 4.02(\mathrm{dd}, J=4.6,10.5 \mathrm{~Hz}, 1 \mathrm{H}), 3.92(\mathrm{dd}, J=4.5,10.5 \mathrm{~Hz}, 1 \mathrm{H}), 3.87$ (d, $J=13.3 \mathrm{~Hz}, 2 \mathrm{H}), 3.60$ (d, $J=13.3 \mathrm{~Hz}, 2 \mathrm{H}), 3.43$ (s, 3H), 3.19 (br s, 1H), 2.99 (dd, $J$ $=2.8,17.4 \mathrm{~Hz}, 1 \mathrm{H}), 2.76(\mathrm{~m}, 1 \mathrm{H}), 2.38(\mathrm{dd}, J=9.2,17.4 \mathrm{~Hz}, 1 \mathrm{H}), 2.08(\mathrm{~s}, 3 \mathrm{H}) .{ }^{13} \mathrm{C}$ NMR (400 MHz, $\left.\mathrm{CDCl}_{3}\right) \delta 210.2,139.9,128.1,128.4,127.2,97.0,67.4,64.9,60.2$, 55.7, 55.3, 48.0, 30.9. FT-IR $v$ (thin film) 3479, 3086, 3063, 3029, 2933, 2888, 2824, 1710, 1603, 1586, 1494, 1454, 1361, 1271, 1211, 1151, $1107 \mathrm{~cm}^{-1}$. EI-MS m/z $340(\mathrm{M}$ - MeO $)^{+}, 296,285,284,181,91,65,43$. HRMS calcd for $\mathrm{C}_{22} \mathrm{H}_{29} \mathrm{NO}_{4}(\mathrm{M}+\mathrm{H})^{+}$requires $m / z$ 372.2176, found $m / z 372.2168$.

(4S,5S)-5-Dibenzylamino-4-hydroxy-6-methoxymethoxyhexan-2-one 8f.

$[\alpha]_{\mathrm{D}}{ }^{16}-43.5\left(c 1.5 \mathrm{CHCl}_{3}\right) .{ }^{1} \mathrm{H} \mathrm{NMR}\left(400 \mathrm{MHz}, \mathrm{CDCl}_{3}\right) \delta 7.36-7.23(\mathrm{~m}, 10 \mathrm{H}), 4.62(\mathrm{~d}$, $J=0.9 \mathrm{~Hz}, 2 \mathrm{H}), 4.17(\mathrm{~m}, 1 \mathrm{H}), 4.04(\mathrm{br} \mathrm{s}, 1 \mathrm{H}), 3.99(\mathrm{~d}, J=13.3 \mathrm{~Hz}, 2 \mathrm{H}), 3.85$ (dd, $J=$ $5.5,10.5 \mathrm{~Hz}, 1 \mathrm{H}), 3.70(\mathrm{dd}, J=5.5,10.5 \mathrm{~Hz}, 1 \mathrm{H}), 3.52(\mathrm{~d}, J=13.3 \mathrm{~Hz}, 2 \mathrm{H}), 3.41(\mathrm{~s}$, 3H), $2.67(\mathrm{~m}, 1 \mathrm{H}), 2.50(\mathrm{~d}, J=6.0 \mathrm{~Hz}, 2 \mathrm{H}), 2.12(\mathrm{~s}, 3 \mathrm{H}) .{ }^{13} \mathrm{C} \mathrm{NMR}\left(400 \mathrm{MHz}, \mathrm{CDCl}_{3}\right)$ $\delta 208.2,139.0,129.3,128.6,127.4,96.8,65.4,63.8,61.5,55.8,54.5,48.1,31.0$.

(R)-2-((1R,2S)-2-Dibenzylamino-1-hydroxy-4-methylpentyl)cyclopentan- 
one 7 g (containing $3 \%$ inseparable isomers 8g). $[\alpha]_{\mathrm{D}}{ }^{17}+36.9\left(c 0.9 \mathrm{CHCl}_{3}\right) .{ }^{1} \mathrm{H}$ NMR (400 MHz, $\mathrm{CDCl}_{3}$ ) (major product 7g) $\delta$ 7.30-7.20 (m, 10H), $4.30(\mathrm{~m}, 1 \mathrm{H}), 3.67$ $(\mathrm{d}, J=13.3 \mathrm{~Hz}, 2 \mathrm{H}), 3.48(\mathrm{~d}, J=13.3 \mathrm{~Hz}, 1 \mathrm{H}), 2.59(\mathrm{~m}, 2 \mathrm{H}), 2.28(\mathrm{~m}, 1 \mathrm{H}), 1.98(\mathrm{~m}$, 1H), 1.89-1.50 (m, 5H), $1.40(\mathrm{~m}, 1 \mathrm{H}), 1.04(\mathrm{~m}, 1 \mathrm{H}), 0.96(\mathrm{~d}, J=6.4 \mathrm{~Hz}, 3 \mathrm{H}), 0.86(\mathrm{~d}, J$ $=6.4 \mathrm{~Hz}, 3 \mathrm{H})$. FT-IR $v$ (thin film) 3519, 3086, 3063, 3029, 2957, 2868, 2804, 1725, $1494,1454,1404,1367,1326,1272,1250,1157,1112,1073,1028 \mathrm{~cm}^{-1}$. ESI-MS m/z $380.3(\mathrm{M}+\mathrm{H})^{+}$. HRMS calcd for $\mathrm{C}_{25} \mathrm{H}_{34} \mathrm{NO}_{2}(\mathrm{M}+\mathrm{H})^{+}$requires $m / z$ 380.2590, found $m / z 380.2567$.

\section{(R)-2-((1R,2S)-2-Dibenzylamino-1-hydroxy-3-phenylpropyl)-cyclopentan-}

one $7 \mathbf{h}$ (containing $2 \%$ inseparable isomers $8 \mathrm{~h}) \cdot[\alpha]_{\mathrm{D}}{ }^{18}+78.2\left(c 1.2 \mathrm{CHCl}_{3}\right) .{ }^{1} \mathrm{H}$ NMR (400 MHz, $\mathrm{CDCl}_{3}$ ) (major product 7h) $\delta$ 7.35-7.14 (m, 15H), $4.39(\mathrm{~m}, 1 \mathrm{H}), 3.75$ $(\mathrm{d}, J=13.3 \mathrm{~Hz}, 2 \mathrm{H}), 3.43(\mathrm{~d}, J=13.3 \mathrm{~Hz}, 2 \mathrm{H}), 3.12(\mathrm{dd}, J=6.4,13.7 \mathrm{~Hz}, 1 \mathrm{H})$, 3.02-2.93 (m, 2H), $2.70(\mathrm{~m}, 1 \mathrm{H}), 2.26(\mathrm{~m}, 1 \mathrm{H}), 1.95(\mathrm{~m}, 1 \mathrm{H}), 1.78(\mathrm{~m}, 1 \mathrm{H}), 1.53-1.42$ (m, 2H), 0.95 (m, 1H). FT-IR $v$ (thin film) 3508, 3086, 3063, 3028, 2963, 2882, 1725 , 1603, 1494, 1454, 1403, 1362, 1251, 1209, 1111, 1074, $1027 \mathrm{~cm}^{-1}$. EI-MS m/z $322(\mathrm{M}$ - Bn $)^{+}, 301,300,184,181,91,84,65$. HRMS calcd for $\mathrm{C}_{28} \mathrm{H}_{31} \mathrm{NO}_{2}(\mathrm{M}+\mathrm{H})^{+}$requires $m / z$ 414.2434, found $m / z 414.2430$.

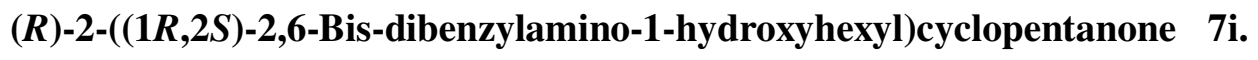
$[\alpha]_{\mathrm{D}}{ }^{16}+50.1\left(c 1.1 \mathrm{CHCl}_{3}\right) .{ }^{1} \mathrm{H}$ NMR $\left(400 \mathrm{MHz}, \mathrm{CDCl}_{3}\right) \delta$ 7.40-7.21 (m, 20H), 4.19 $(\mathrm{dd}, J=0.9,8.7 \mathrm{~Hz}, 1 \mathrm{H}), 3.64(\mathrm{~d}, J=13.3 \mathrm{~Hz}, 2 \mathrm{H}), 3.59(\mathrm{~d}, J=13.7 \mathrm{~Hz}, 2 \mathrm{H}), 3.50(\mathrm{~d}, J$ $=13.7 \mathrm{~Hz}, 2 \mathrm{H}), 3.41(\mathrm{~d}, J=13.3 \mathrm{~Hz}, 2 \mathrm{H}), 2.59(\mathrm{~m}, 1 \mathrm{H}), 2.45(\mathrm{~m}, 3 \mathrm{H}), 2.25(\mathrm{~m}, 1 \mathrm{H})$, $1.98(\mathrm{~m}, 3 \mathrm{H}), 1.81(\mathrm{~m}, 1 \mathrm{H}), 1.67-1.37(\mathrm{~m}, 5 \mathrm{H}), 1.26(\mathrm{~m}, 1 \mathrm{H}), 0.90(\mathrm{~m}, 1 \mathrm{H}) .{ }^{13} \mathrm{C} \mathrm{NMR}$ 
$\left(400 \mathrm{MHz}, \mathrm{CDCl}_{3}\right) \delta 222.7,140.1,140.0,129.5,129.1,129.0,128.3,127.1,126.9$, 70.8, 59.3, 58.5, 54.5, 53.1, 53.0, 39.1, 27.6, 26.6, 26.5, 21.9, 20.6. FT-IR $v$ (thin film) $3518,3086,3063,3028,2939,2800,1725,1602,1586,1494,1453,1403,1371,1326$, 1248, 1155, 1114, 1074, $1028 \mathrm{~cm}^{-1}$. ESI-MS m/z $575.4(\mathrm{M}+\mathrm{H})^{+}$. HRMS calcd for $\mathrm{C}_{39} \mathrm{H}_{46} \mathrm{~N}_{2} \mathrm{O}_{2}(\mathrm{M}+\mathrm{H})^{+}$requires $m / z, 575.3638$, found $m / z$ 575.3624.

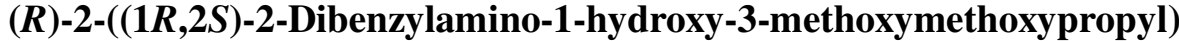

cyclopentanone $7 \mathbf{j} \cdot[\alpha]_{\mathrm{D}}{ }^{17}+83.9\left(c 1.0 \mathrm{CHCl}_{3}\right) .{ }^{1} \mathrm{H}$ NMR $\left(400 \mathrm{MHz}, \mathrm{CDCl}_{3}\right) \delta$ 7.33-7.22 (m, 10H), $4.68(\mathrm{dd}, J=6.4,8.2 \mathrm{~Hz}, 2 \mathrm{H}), 4.47(\mathrm{~m}, 1 \mathrm{H}), 4.05(\mathrm{dd}, J=4.6,10.1$ Hz, 1H), $3.94(\mathrm{dd}, J=4.6,10.1 \mathrm{~Hz}, 1 \mathrm{H}), 3.91(\mathrm{~d}, J=13.3 \mathrm{~Hz}, 2 \mathrm{H}), 3.43(\mathrm{~d}, J=13.3 \mathrm{~Hz}$, 2H), $3.43(\mathrm{~s}, 3 \mathrm{H}), 2.73(\mathrm{~m}, 1 \mathrm{H}), 2.67(\mathrm{~m}, 1 \mathrm{H}), 2.59$ (br s, 1H), $2.27(\mathrm{~m}, 1 \mathrm{H}), 2.04(\mathrm{~m}$, 1H), $1.82(\mathrm{~m}, 1 \mathrm{H}), 1.51(\mathrm{~m}, 2 \mathrm{H}), 0.84(\mathrm{~m}, 1 \mathrm{H}) .{ }^{13} \mathrm{C} \mathrm{NMR}\left(400 \mathrm{MHz}, \mathrm{CDCl}_{3}\right) \delta 222.2$ $139.7,129.4,128.4,127.2,97.2,69.4,65.1,58.3,55.9,55.0,51.9,39.3,21.3,20.7$. FT-IR $v$ (thin film) 3461, 3086, 3063, 3029, 2939, 2884, 1732, 1668, 1603, 1495, 1454, $1405,1273,1254,1212,1153,1111,1075,1043 \mathrm{~cm}^{-1}$. EI-MS m/z $366(\mathrm{M}-\mathrm{MeO})^{+}$, $322,304,285,284,181,91,83,65,45$. HRMS calcd for $\mathrm{C}_{24} \mathrm{H}_{32} \mathrm{NO}_{4}(\mathrm{M}+\mathrm{H})^{+}$requires $m / z$ 398.2332, found $m / z 398.2323$.

\section{$(3 S, 4 S, 5 S)-5-D i b e n z y l a m i n o-3,4-d i h y d r o x y-6-p h e n y l h e x a n-2-o n e \quad 7 k$.}

$[\alpha]_{\mathrm{D}}{ }^{16}+31.6\left(c 1.0 \mathrm{CHCl}_{3}\right) .{ }^{1} \mathrm{H} \mathrm{NMR}\left(400 \mathrm{MHz}, \mathrm{CDCl}_{3}\right) \delta 7.36-7.18(\mathrm{~m}, 15 \mathrm{H}), 3.88(\mathrm{~d}$, $J=13.3 \mathrm{~Hz}, 2 \mathrm{H}), 3.84(\mathrm{~m}, 2 \mathrm{H}), 3.51(\mathrm{~d}, J=13.3 \mathrm{~Hz}, 2 \mathrm{H}), 3.30(\mathrm{dd}, J=7.4,12.8 \mathrm{~Hz}$ 1H), 3.22-3.10 (m, 2H), $2.15(\mathrm{~s}, 3 \mathrm{H}) .{ }^{13} \mathrm{C} \mathrm{NMR}\left(400 \mathrm{MHz}, \mathrm{CDCl}_{3}\right) \delta$ 212.7, 141.2, 138.3, 129.7, 129.5, 128.6, 127.6, 126.2, 79.8, 71.3, 63.7, 54.9, 32.1, 27.2. FT-IR v (thin film) 3449, 3086, 3063, 3028, 2929, 2805, 1710, 1664, 1603, 1585, 1495, 1454, 
1357, 1210, 1120, 1071, $1029 \mathrm{~cm}^{-1}$. EI-MS m/z 360 (M - Ac) ${ }^{+}, 312,301,300,238,181$, 91, 65, 43. HRMS calcd for $\mathrm{C}_{26} \mathrm{H}_{29} \mathrm{NO}_{3}(\mathrm{M}+\mathrm{H})^{+}$requires $\mathrm{m} / \mathrm{z}$ 404.2226, found $\mathrm{m} / \mathrm{z}$ 404.2228 .

(3S,4S,5S)-5-Dibenzylamino-3,4-dihydroxyhexan-2-one $7 \mathrm{~m}$ (containing 4\% inseparable isomers). $[\alpha]_{\mathrm{D}}{ }^{18}+17.9\left(c^{1.1} \mathrm{CHCl}_{3}\right) .{ }^{1} \mathrm{H} \mathrm{NMR}\left(400 \mathrm{MHz}, \mathrm{CDCl}_{3}\right)$ (major product $7 \mathbf{m}) \delta 7.36-7.26(\mathrm{~m}, 10 \mathrm{H}), 3.87(\mathrm{~d}, J=13.3 \mathrm{~Hz}, 2 \mathrm{H}), 3.76(\mathrm{dd}, J=7.8$, $9.6 \mathrm{~Hz}, 1 \mathrm{H}), 3.66(\mathrm{~d}, J=7.8 \mathrm{~Hz}, 1 \mathrm{H}), 3.35(\mathrm{~d}, J=13.3 \mathrm{~Hz}, 2 \mathrm{H}), 3.04(\mathrm{~m}, 1 \mathrm{H}), 2.23(\mathrm{~s}$, 3H), 1.24 (d, $J=6.9 \mathrm{~Hz}, 3 \mathrm{H})$. FT-IR v (thin film) 3342, 3086, 3063, 3028, 2968, 2912, $2850,1725,1602,1585,1496,1452,1232,1176,1140,1110,1068,1026,1004 \mathrm{~cm}^{-1}$. EI-MS $m / z, 284(\mathrm{M}-\mathrm{Ac})^{+}, 224,181,132,91,65,43 . \mathrm{HRMS}$ calcd for $\mathrm{C}_{20} \mathrm{H}_{26} \mathrm{NO}_{3}(\mathrm{M}+$ $\mathrm{H})^{+}$requires $m / z$ 328.1913, found $m / z$ 328.1916.

(3S,4S,5S)-5-Dibenzylamino-3,4-dihydroxy-7-methyl-octan-2-one 7 . $[\alpha]_{\mathrm{D}}{ }^{17}+19.4\left(c 1.0 \mathrm{CHCl}_{3}\right) .{ }^{1} \mathrm{H} \mathrm{NMR}\left(400 \mathrm{MHz}, \mathrm{CDCl}_{3}\right) \delta$ 7.35-7.23 (m, 10H), $3.86(\mathrm{~m}$, 2H), $3.80(\mathrm{~d}, J=13.3 \mathrm{~Hz}, 2 \mathrm{H}), 3.52(\mathrm{~d}, J=13.3 \mathrm{~Hz}, 2 \mathrm{H}), 2.95(\mathrm{~m}, 1 \mathrm{H}), 2.18(\mathrm{~s}, 3 \mathrm{H})$, $1.96(\mathrm{~m}, 1 \mathrm{H}), 1.59(\mathrm{~m}, 2 \mathrm{H}), 0.95(\mathrm{~d}, J=6.8 \mathrm{~Hz}, 3 \mathrm{H}), 0.88(\mathrm{~d}, J=6.8 \mathrm{~Hz}, 3 \mathrm{H}) .{ }^{13} \mathrm{C}$ NMR $\left(400 \mathrm{MHz}, \mathrm{CDCl}_{3}\right) \delta 213.1,138.7,129.5,128.6,127.5,79.8,72.0,59.3,54.5$, 35.8, 27.3, 26.1, 23.5, 22.9. FT-IR $v$ (thin film) 3450, 3087, 3064, 3030, 2956, 2868, 2806, 1710, 1603, 1495, 1454, 1357, 1209, 1105, 1068, $1029 \mathrm{~cm}^{-1}$. EI-MS m/z $326(\mathrm{M}$ - Ac) $)^{+}, 267,266,181,91,65,43$. HRMS calcd for $\mathrm{C}_{23} \mathrm{H}_{31} \mathrm{NO}_{3}(\mathrm{M}+\mathrm{H})^{+}$requires $m / z$ 370.2383 , found $m / z 370.2364$.

$(3 S, 4 S, 5 S)-5,9-B i s-d i b e n z y l a m i n o-3,4-d i h y d r o x y n o n a n-2-o n e \quad 7 o \cdot[\alpha]_{D}^{18}$ $+25.8\left(c 1.0 \mathrm{CHCl}_{3}\right) .{ }^{1} \mathrm{H}$ NMR $\left(400 \mathrm{MHz}, \mathrm{CDCl}_{3}\right) \delta 7.40-7.17(\mathrm{~m}, 20 \mathrm{H}), 3.79(\mathrm{~d}, J=$ 
$13.3 \mathrm{~Hz}, 2 \mathrm{H}), 3.74(\mathrm{~m}, 2 \mathrm{H}), 3.56(\mathrm{dd}, J=13.3,23.8 \mathrm{~Hz}, 4 \mathrm{H}), 3.43(\mathrm{~d}, J=13.3 \mathrm{~Hz}, 2 \mathrm{H})$, $2.84(\mathrm{~m}, 1 \mathrm{H}), 2.46(\mathrm{~m}, 2 \mathrm{H}), 2.17(\mathrm{~s}, 3 \mathrm{H}), 1.55(\mathrm{~m}, 6 \mathrm{H}) .{ }^{13} \mathrm{C} \mathrm{NMR}\left(400 \mathrm{MHz}, \mathrm{CDCl}_{3}\right) \delta$ $213.4,140.0,138.3,129.6,129.0,128.7,128.3,127.6,126.9,80.1,71.2,61.7,58.5$ 54.6, 53.1, 27.7, 27.2, 26.6, 26.0. FT-IR $v$ (thin film) 3449, 3086, 3063, 3028, 2935, 2799, 1709, 1602, 1494, 1453, 1357, 1243, 1126, 1074, $1029 \mathrm{~cm}^{-1}$. ESI-MS m/z 565.3 $(\mathrm{M}+\mathrm{H})^{+}$. HRMS calcd for $\mathrm{C}_{37} \mathrm{H}_{45} \mathrm{~N}_{2} \mathrm{O}_{3}(\mathrm{M}+\mathrm{H})^{+}$requires $\mathrm{m} / z$ 565.3431, found $\mathrm{m} / z$ 565.3413.

(3S,4S,5S)-5-Dibenzylamino-3,4-dihydroxy-6-methoxymethoxy-hexan-2one $7 p$ (containing $9 \%$ inseparable isomers). $[\alpha]_{\mathrm{D}}{ }^{17}+43.4\left(c 1.6 \mathrm{CHCl}_{3}\right) .{ }^{1} \mathrm{H} \mathrm{NMR}$ $\left(400 \mathrm{MHz}, \mathrm{CDCl}_{3}\right)$ (major product 7p) $\delta$ 7.36-7.21 (m, 10H), $4.70(\mathrm{~s}, 2 \mathrm{H}), 4.09-3.93$ (m, 3H), $4.00(\mathrm{~d}, J=13.3 \mathrm{~Hz}, 2 \mathrm{H}), 3.81(\mathrm{~d}, J=6.9 \mathrm{~Hz}, 1 \mathrm{H}), 3.59,(\mathrm{~d}, J=13.3 \mathrm{~Hz}, 2 \mathrm{H})$, $3.45(\mathrm{~s}, 3 \mathrm{H}), 3.15(\mathrm{~m}, 1 \mathrm{H}), 2.14$ (s, 3H). FT-IR v (thin film) 3447, 3086, 3063, 3031, $2886,1709,1603,1495,1454,1359,1211,1145,1111,1048,1025 \mathrm{~cm}^{-1}$. EI-MS m/z $356(\mathrm{M}-\mathrm{MeO})^{+}, 312,284,181,91,65,45$. HRMS calcd for $\mathrm{C}_{22} \mathrm{H}_{30} \mathrm{NO}_{5}(\mathrm{M}+\mathrm{H})^{+}$ requires $m / z$ 388.2125, found $m / z$ 388.2125.

(2S,3R,5S)-2-Benzyl-5-methylpyrrolidin-3-ol 4. A solution of $2 \mathrm{~d}$ (180 mg, $0.47 \mathrm{mmol})$, in methanol (5 mL) was hydrogenated with $10 \% \mathrm{Pd} / \mathrm{C}(60 \mathrm{mg})$ under 1 atm for $24 \mathrm{~h}$ at room temperature. After $\mathrm{Pd} / \mathrm{C}$ was filtered off, the filtrate was concentrated and the residue was purified by flash chromatography with methanol/ethyl acetate mixtures to give $49 \mathrm{mg}$ of $\mathbf{4}$ (55\% yield) and $20 \mathrm{mg}$ of $\mathbf{5}$ (23\% yield). $\quad[\alpha]_{\mathrm{D}}{ }^{20}-45.7(c 0.7 \mathrm{MeOH}) .{ }^{1} \mathrm{H} \mathrm{NMR}\left(400 \mathrm{MHz}, \mathrm{CDCl}_{3}\right) \delta$ 7.31-7.19 (m, 5H), $4.04(\mathrm{~m}, 1 \mathrm{H}), 3.38(\mathrm{~m}, 2 \mathrm{H}), 3.30$ (brs, 2H), $2.75(\mathrm{~d}, J=7.3 \mathrm{~Hz}, 2 \mathrm{H}), 2.36(\mathrm{~m}, 1 \mathrm{H}), 1.42$ 
$(\mathrm{m}, 1 \mathrm{H}), 1.22(\mathrm{~d}, J=6.4 \mathrm{~Hz}, 3 \mathrm{H}) .{ }^{13} \mathrm{C} \mathrm{NMR}\left(400 \mathrm{MHz}, \mathrm{CDCl}_{3}\right) \delta 138.6,129.2,128.8$, 126.6, 76.3, 67.0, 51.4, 42.1, 39.0, 22.3. FT-IR $v$ (thin film) 3352, 3296, 3086, 3060, 2966, 2833, 1497, 1453, 1415, 1377, 1125, 1088, 1070, $1008 \mathrm{~cm}^{-1}$. EI-MS m/z $192(\mathrm{M}$ $+\mathrm{H})^{+}, 132,104,100,91,82,77,65,44 . \mathrm{HRMS}$ calcd for $\mathrm{C}_{12} \mathrm{H}_{17} \mathrm{NO}(\mathrm{M}+\mathrm{H})^{+}$requires $m / z$ 192.1389, found $m / z 192.1378$.

\section{$(2 S, 3 R, 3 a S, 6 a R)$-2-Methoxymethoxymethyl-octahydro-cyclopenta[b]-}

pyrrol-3-ol 9. A solution of $7 \mathbf{j}$ (180 $\mathrm{mg}, 0.45 \mathrm{mmol})$, in methanol $(5 \mathrm{~mL})$ was hydrogenated with $10 \% \mathrm{Pd} / \mathrm{C}(60 \mathrm{mg})$ under $1 \mathrm{~atm}$ for $72 \mathrm{~h}$ at room temperature. After $\mathrm{Pd} / \mathrm{C}$ was filtered off, the filtrate was concentrated and the residue was purified by flash chromatography with methanol/ethyl acetate mixtures to give $62 \mathrm{mg}$ of $\mathbf{9}(68 \%$

yield). $[\alpha]_{\mathrm{D}}{ }^{20}-7.0(c 1.0 \mathrm{MeOH}) .{ }^{1} \mathrm{H} \mathrm{NMR}\left(400 \mathrm{MHz}, \mathrm{CDCl}_{3}\right) \delta 4.64(\mathrm{~s}, 2 \mathrm{H}), 3.84(\mathrm{~m}$, 1H), $3.68(\mathrm{~m}, 2 \mathrm{H}), 3.53(\mathrm{~m}, 1 \mathrm{H}), 3.37(\mathrm{~s}, 3 \mathrm{H}), 3.05(\mathrm{~m}, 1 \mathrm{H}), 2.58$ (brs, $2 \mathrm{H}), 2.40(\mathrm{~m}$, 1H), 1.73-1.48 (m, 6H). ${ }^{13} \mathrm{C}$ NMR (400 MHz, $\left.\mathrm{CDCl}_{3}\right) \delta$ 96.9, 81.2, 69.1, 64.7, 61.6, 55.5, 51.5, 34.1, 31.2, 24.3. ESI-MS $m / z 202.1(\mathrm{M}+\mathrm{H})^{+}$. HRMS calcd for $\mathrm{C}_{10} \mathrm{H}_{20} \mathrm{NO}_{3}$ $(\mathrm{M}+\mathrm{H})^{+}$requires $m / z$ 202.1444, found $m / z$ 202.1434.

(2S, 3S, 4R, 5S)-2-Benzyl-5-methyl-pyrrolidine-3, 4-diol 10. A solution of 7k (180 mg, $0.45 \mathrm{mmol})$, in methanol $(5 \mathrm{~mL})$ was hydrogenated with $10 \% \mathrm{Pd} / \mathrm{C}$ (60 $\mathrm{mg}$ ) under $1 \mathrm{~atm}$ for $72 \mathrm{~h}$ at room temperature. After $\mathrm{Pd} / \mathrm{C}$ was filtered off, the filtrate was concentrated and the residue was purified by flash chromatography with methanol/ethyl acetate mixtures to give $63 \mathrm{mg}$ of $\mathbf{1 0}\left(68 \%\right.$ yield). $[\alpha]_{\mathrm{D}}{ }^{20}-55.7$ (c 0.6 MeOH). ${ }^{1} \mathrm{H}$ NMR (400 MHz, $\left.\mathrm{CDCl}_{3}\right) \delta$ 7.32-7.20 (m, 5H), $3.87(\mathrm{~m}, 2 \mathrm{H}), 3.32(\mathrm{~m}, 1 \mathrm{H})$, $3.24(\mathrm{~m}, 1 \mathrm{H}), 2.90(\mathrm{dd}, J=5.5,13.7 \mathrm{~Hz}, 1 \mathrm{H}), 2.75(\mathrm{br} \mathrm{s}, 3 \mathrm{H}), 2.72(\mathrm{dd}, J=8.2,13.7 \mathrm{~Hz}$, 
$1 \mathrm{H}), 1.12(\mathrm{~d}, J=6.4 \mathrm{~Hz}, 3 \mathrm{H}) .{ }^{13} \mathrm{C} \mathrm{NMR}\left(400 \mathrm{MHz}, \mathrm{CDCl}_{3}\right) \delta$ 139.0, 129.2, 128.7, 126.5, 78.1, 74.0, 63.5, 54.7, 40.7, 15.0. EI-MS m/z $208(\mathrm{M}+\mathrm{H})^{+}, 160,132,120,116$, 104, 91, 77, 57, 44. HRMS calcd for $\mathrm{C}_{12} \mathrm{H}_{17} \mathrm{NO}_{2}(\mathrm{M}+\mathrm{H})^{+}$requires $\mathrm{m} / z$ 208.1338, found $m / z 208.1335$.

\section{(2S,3S,4S)-4-Dibenzylamino-2,3-dihydroxy-6-methylheptanoic acid benzyl}

ester 11. To a sodium hypobromite solution (prepared from $600 \mathrm{mg}(3.75 \mathrm{mmol})$ of bromine and $420 \mathrm{mg}(10.5 \mathrm{mmol})$ of sodium hydroxide (4 $\mathrm{mL}$ of water)) was added slowly the solution of $370 \mathrm{mg}(1 \mathrm{mmol})$ of $\mathbf{7 n}$ in $0.4 \mathrm{~mL}$ of dioxane. The mixture was stirred in an ice bath for $1 \mathrm{~h}$ and then overnight at $10{ }^{\circ} \mathrm{C}$. The solution was treated with solid sodium bisulfite until the test for excess hypobromite with acidified potassium iodide solution was negative (a positive color was brown). Then the mixture was acidified to $\mathrm{pH} 6$ with $4 \mathrm{M} \mathrm{HCl}$ and extracted with ether two times. The combined organic layers were washed with brine, dried over $\mathrm{Na}_{2} \mathrm{SO}_{4}$, and concentrated. The crude product was dissolved in $5 \mathrm{~mL}$ DMF and to the solution was added $\mathrm{K}_{2} \mathrm{CO}_{3}$ (138 $\mathrm{mg}, 1 \mathrm{mmol})$ and $\mathrm{BnBr}(0.24 \mathrm{~mL}, 2 \mathrm{mmol})$. The mixture was stirred overnight and extracted with ethyl acetate two times. The combined organic layer was washed with water and brine, dried over $\mathrm{Na}_{2} \mathrm{SO}_{4}$, filtered, and concentrated. The resulting residue was then purified by silica gel chromatography eluting with hexane/ethyl acetate (4:1) to give $161 \mathrm{mg}$ of 11 (35\% yield). [ $\alpha]_{\mathrm{D}}{ }^{20}-4.6\left(c 0.9 \mathrm{CHCl}_{3}\right) .{ }^{1} \mathrm{H} \mathrm{NMR}(400 \mathrm{MHz}$, $\left.\mathrm{CDCl}_{3}\right) \delta 7.37-7.20(\mathrm{~m}, 15 \mathrm{H}), 5.17(\mathrm{~d}, J=11.9 \mathrm{~Hz}, 1 \mathrm{H}), 5.10(\mathrm{~d}, J=11.9 \mathrm{~Hz}, 1 \mathrm{H}), 4.15$ $(\mathrm{d}, J=6.9 \mathrm{~Hz}, 1 \mathrm{H}), 4.00(\mathrm{dd}, J=6.4,6.9 \mathrm{~Hz}, 1 \mathrm{H}), 3.70(\mathrm{~d}, J=13.3 \mathrm{~Hz}, 2 \mathrm{H}), 3.57(\mathrm{~d}, J$ $=13.3 \mathrm{~Hz}, 2 \mathrm{H}), 2.99(\mathrm{~m}, 1 \mathrm{H}), 1.89(\mathrm{~m}, 1 \mathrm{H}), 1.61(\mathrm{~m}, 1 \mathrm{H}), 1.39(\mathrm{~m}, 1 \mathrm{H}), 0.88(\mathrm{~d}, J=$ 
$6.4 \mathrm{~Hz}, 3 \mathrm{H}), 0.76(\mathrm{~d}, J=6.4 \mathrm{~Hz}, 3 \mathrm{H}) .{ }^{13} \mathrm{C} \mathrm{NMR}\left(400 \mathrm{MHz}, \mathrm{CDCl}_{3}\right) \delta 173.4,139.2$, $135.2,129.4,128.7,128.60,128.56,128.4,127.3,74.3,72.6,67.4,57.9,54.5,35.8$, 25.9, 23.2, 23.0. ESI-MS $m / z 462.3(\mathrm{M}+\mathrm{H})^{+}$. HRMS calcd for $\mathrm{C}_{29} \mathrm{H}_{35} \mathrm{NO}_{4}(\mathrm{M}+\mathrm{H})^{+}$ requires $m / z$ 462.2645, found $m / z$ 462.2631. 